\title{
DESIGN OF A SUPERCONDUCTING MAGNETIC SUSPENSION SYSTEM FOR A LIQUID HELIUM FLOW EXPERIMENT
}

\author{
Michael R. Smith, Yehia M. Eyssa, and Steven W. Van Sciver \\ National High Magnetic Field Laboratory \\ Tallahassee, FL
}

\section{SUMMARY}

We discuss a preliminary design for a superconducting magnetic suspension system for measurement of drag on rotationally symmetric bodies in liquid helium. Superconducting materials are a natural choice for liquid helium studies, since temperatures are well below most critical temperatures, so that the resulting heat load is negligible. Also, due to its diamagnetic properties, a superconducting model (for example made or coated with $\mathrm{Nb}$ ) is inherently stable against disturbances. Issues which we consider include model placement during initial cool-down, maintaining placement during anticipated drag and lift forces, and force measurement. This later can be achieved by a passive technique, where the body is allowed to deflect under the influence of drag from its neutral position. The resulting shift in flux is detected via a superconducting pickup coil. The pickup coil may be connected either to a SQUID, or a secondary loop wound around a Hall probe. Both options are discussed. The objective of this work is to gain a better understanding of the nature of turbulent fields in normal and superfluid helium for potential application to problems in classical high Reynolds number turbulence.

\section{INTRODUCTION}

The design and construction of non-intrusive force balance systems has long been an important aspect of dynamic studies carried out in wind tunnels. Early research concentrated on understanding the interaction of physical struts or stings with the flow field under examination, in an effort to subtract these effects for the relevant measurements ${ }^{1}$. With the advent of Magnetic Suspension and Balance Systems (MSBS), the problem of physical intrusion into the flow field has been eliminated at the cost of a formidable controls problem ${ }^{2,3,4}$. Simplification may come as a by-product of using liquid helium as a test fluid. Because its kinematic viscosity is over 800 times smaller than that of air, liquid helium offers the potential of extending the range of Reynolds numbers available in wind tunnel testing of high performance aircraft and marine vessels ${ }^{5}$. Liquid helium also offers operating temperatures well below the critical temperature of most superconductors, thus allowing a superconducting MSBS. Such a system offers several advantages over its resistive counterparts. First, a superconducting MSBS dissipates no heat resistively, and so may be operated in the helium bath. Operated in persistent mode (as a closed system disconnected from external supplies and noise sources), such a 'passive' MSBS may be constructed, as compared with the active systems traditionally built. A passive MSBS eliminates the need for continuous 
control/feedback and reduces noise. A superconducting system is then potentially quieter and more sensitive. Second, superconductors in a magnetic field behave as strongly diamagnetic materials which reject flux, and so are repelled by regions of high magnetic field intensity. A superconducting MSBS would then have improved coupling between the model and support field and be inherently stable, again improving sensitivity and simplifying the control problem. Finally, since flux through a superconducting loop in persistent operation is perfectly conserved, one may envision a completely magnetic MSBS, without resorting to optical position sensing schemes. With the MSBS coils operated in persistent mode, and the body is allowed to deflect with applied drag from its neutral or 'no flow' position. The resulting flux shift could then be detected with a superconducting loop wound around the test section. The degree to which this may be carried out depends upon the symmetry of the body and flow field.

In this paper, we consider the design of a superconducting MSBS for supporting and measuring the drag on a sphere in a liquid helium flow field. Current levels and signal output are presented, together with a discussion of certain practical issues which must be considered in the actual construction and operation of a prototype. The optimum design was selected to take advantage of existing facilities at the National High Magnetic Field Laboratory at Florida State University.

\section{GENERAL CONSIDERATIONS}

As a first step, we need to estimate the scale of the experiment together with anticipated forces. A conservative estimate of the maximum mass flow available with our equipment is $\dot{m} \approx 50 \mathrm{~g} / \mathrm{sec}$, so long as the pressure drop across the test section is not too large. For fixed mass flow, the drag and Reynolds number vary as

$$
\begin{aligned}
& \mathrm{F}_{\mathrm{I}}=\frac{2 \dot{m}^{2} \mathrm{C}_{\mathrm{D}}}{\pi \rho \mathrm{d}_{\mathrm{s}}^{2}\left(A^{2}-1\right)^{2}} \\
& \operatorname{Re}=\frac{4 \dot{m}}{\pi \rho v \mathrm{~d}_{\mathrm{s}}\left(A^{2}-1\right)}
\end{aligned}
$$

where $\mathrm{d}_{\mathrm{s}}$ is the diameter of the sphere, and $A$ is an aspect ratio $\left(A \equiv \mathrm{d}_{\text {tunnel }} / \mathrm{d}_{\mathrm{s}}\right)$. For a fixed mass flow rate, the largest drag and Reynolds number are achieved for a small $A$ and $\mathrm{d}_{\mathrm{s}}$ (note that velocity is not constant in this situation). The coefficient of drag $C_{D} \sim 0.4$ over the range of Reynolds numbers considered here, and $\rho$ and $v$ are the density and kinematic viscosity, respectively. If the body being tested takes up too much space within the test section, the streamlines no longer approximate their behavior in an infinite flow field. The velocity around the model increases due to the reduced flow area associated with the presence of the model within the tunnel. Pressure, drag, and other forces all exhibit anomalous behavior attributed to this 'blockage'. To minimize blockage effects, most wind tunnel users try to maintain $A \geq 10$. Blockage effects however, are manageable for $A=4$, and the corresponding drag is boosted by a factor of $(99 / 15)^{2}=43.56$. Additionally, we will soon measure drag on a sphere with $A=4$ by other means, and this will serve as a useful point for evaluating the performance of the superconducting MSBS. With $A=4$, this leaves $d_{s}$ as the defining scale of the experiment. From the beginning, in order to save weight, we chose to use a glass or quartz sphere with a thin $\mathrm{Nb}$ film applied to the outside (as opposed to solid superconductor). Making $\mathrm{d}_{\mathrm{s}}$ too small not only makes it difficult to apply such a film, it also increases the 
pressure drop over the test section $\left(\mathrm{d}_{\text {tunnel }}=A \cdot \mathrm{d}_{\mathrm{s}}\right.$ ) and leads to problems in detecting the sphere's position during the experiment. In an effort not to make things too difficult, we chose an easily obtainable $\mathrm{d}_{\mathrm{s}}=9.525 \mathrm{~mm}(0.375 \mathrm{in})$. Then the maximum drag and Reynolds number are of order $2.15 \times 10^{-4} \mathrm{~N}$ and $3 \times 10^{5}$, respectively. A glass sphere of this size will weigh approximately $10^{-2} \mathrm{~N}$, though a heavier sphere of solid niobium may be required if the plating process proves too difficult. In all subsequent calculations, we assume that such a sphere acts as a perfect diamagnet.

With an estimate of the forces in hand, one may consider whether to orient the experiment horizontally or vertically. In the vertical configuration, the weight and flow/drag are co-axial. While this appears to be a simplification, it places the experimentalist in the position of accurately resolving a small fluctuating signal against a large dc background to better than 1 part in $10^{4}$. Further, it is difficult to imagine how one might calibrate such a system or recover in the event of the MSBS 'losing its grip' on the sphere (hereafter called 'blow-by'). In the horizontal configuration however, the drag and support fields are orthogonal, so that sensitive drag measurements may be made with minimal interference from the support coils.

The final issue which had to be resolved up front was whether to use active feedback or a passive system for measuring the drag. In the case of a conventional ferromagnetic MSBS, the support field is attractive by nature, and thus is fundamentally unstable. An active system continuously senses the model's position and makes corrections in the support field to maintain the neutral position. These corrections are calibrated and then read off as forces and moments. Such a system is indispensable for a conventional MSBS. In the passive system, one operates all the superconducting coils in the persistent mode, and the diamagnetic material in or on the model is repelled to the point of lowest field intensity. This system is fundamentally stable, as well as being free from sources of instrument noise in the lab. The sphere deflects slightly from its neutral position under applied drag. This re-distributes the flux in the test section, which may be detected by a superconducting sensing coil around the test section. Having selected the passive approach for the benefits discussed in the introduction, we designed the drag coils as a Helmholtz pair with a linear drag/displacement response over the anticipated range of drag. The field produced by this pair should be reasonably homogeneous over the region which the sphere is allowed to deflect, with the maximum allowed field kept well below the critical field at which superconductivity breaks down $\left(B_{c} \sim 0.15\right.$ $\mathrm{T}$ for $\mathrm{Nb}$ ).

\section{SUPPORT AND DRAG COIL DESIGN}

The general configuration selected is illustrated in Figure 1, which shows cross-sections of the flow experiment together with the coil components. The vertical support coils are of a counterwound racetrack configuration designed to produce a quadrapole field in the plane perpendicular to the flow. Higher current in the coils results in a stiffer field, but also a higher maximum field intensity to which the sphere is exposed. The sphere then displaces slightly under the influence of its weight. This vertical displacement is plotted against the applied field strength in Figure 2, where the radius of the windings was taken to be $43 \mathrm{~mm}$. Since we wish to limit the total maximum field which the sphere experiences (including the contribution from the drag coils, discussed below) to below the critical field, the applied field strength $\mathrm{B}_{\mathrm{s}}=0.018 \mathrm{~T}$ was selected, requiring a total $8.68 \mathrm{kA}$ turns flowing around each coil. The corresponding vertical displacement of the sphere within the field is $1 \mathrm{~mm}$. The associated maximum field on the surface of the sphere was $B_{s, \max }=2.5 \cdot B_{s}=0.045 \mathrm{~T}$. Thus, one should offset the support coils $1 \mathrm{~mm}$ with respect to 


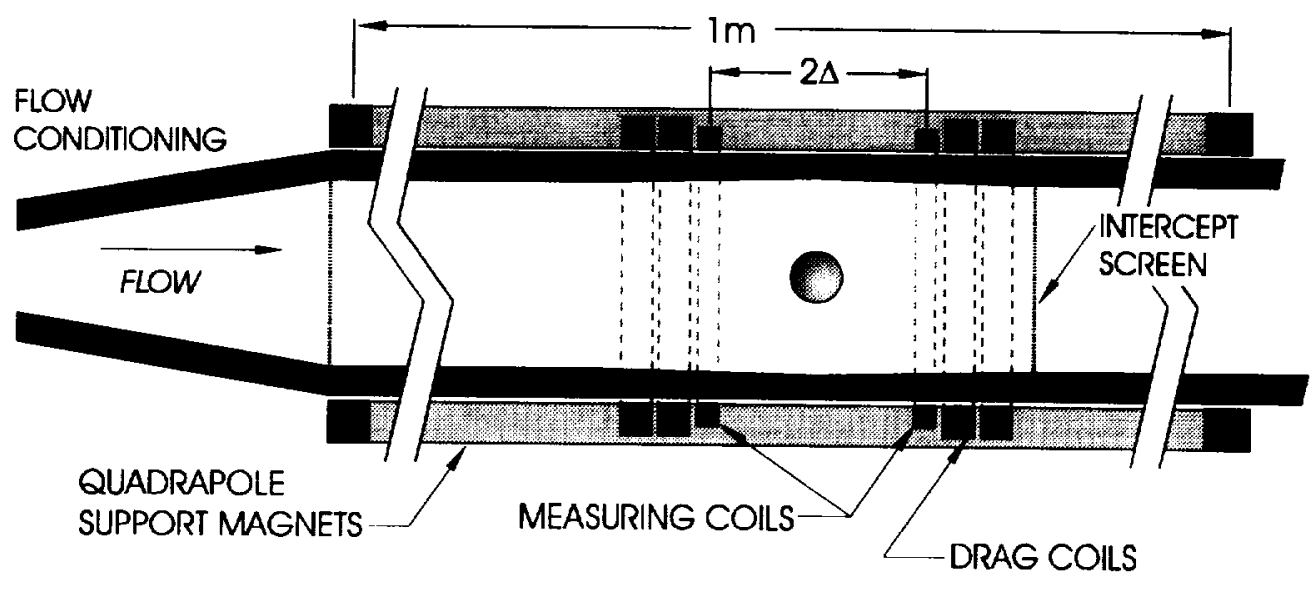

(a)

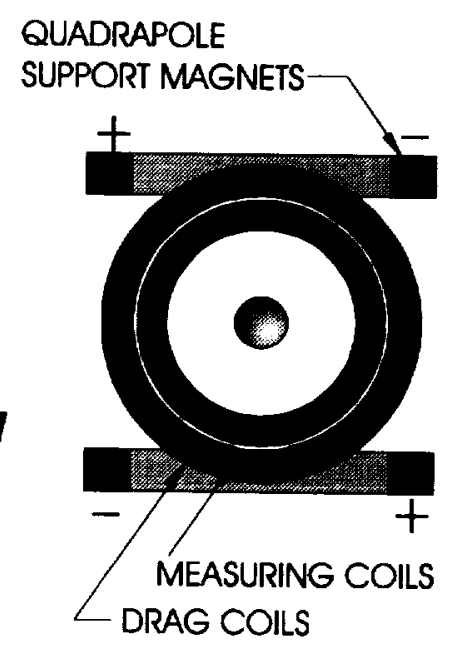

(b)

Figure 1. Schematic of the proposed Superconducting MSBS. Support is accomplished by means of two independent magnet assemblies operated in persistent mode. The support coils are of a counterwound race track configuration, designed to produce a quadrapole field. The drag coil assembly is composed of sets of Helmholtz coils, tuned to provide a particular $\mathrm{B}_{0}$ and $\mathrm{dB} / \mathrm{dz}$. The screen located down stream of the neutral position intercepts the sphere in the event of blow by. The walls form a very slight conical taper, extending up and down stream from the neutral position. This provides a passive mechanism for controlling the location of the sphere without the MSBS energized.

the test section in order to maintain the sphere in the center of the flow field. Figure 3 shows the field lines around the superconducting sphere in a quadrapole field. The plane of this illustration is perpendicular to the flow as in Figure 1b. Arrows point to the regions of highest field intensity.

The drag coils are built up from Helmholtz pairs, designed to produce a particular applied field $\mathrm{B}_{0}$ and field gradient $\mathrm{dB} / \mathrm{dz}$ along the direction of $\mathrm{drag} / \mathrm{flow}$. In principle, a system of coils may be designed to produce any $B_{0}$ and $d B / d z$ desired, so we will not dwell on the details. If the sphere is allowed to deflect a distance $\delta$ under the influence of drag, the restoring force (drag) may be expressed

$$
F_{D}=\frac{2 \pi d_{s}^{3}}{\mu_{0}} B_{o} \frac{d B}{d z}
$$

where $d B / d z$, and therefore $F_{D}$, varies linearly with the displacement. Figure 4 shows a plot of $B_{0} v s$. $d B / d z$ for various values of $F_{D}$. In measuring drag (as discussed in the next section), the optimum signal is achieved for $\mathrm{dB} / \mathrm{dz}$ as small as possible, or equivalently $\mathrm{B}_{0}$ as large as possible, while maximizing the displacement $\delta$ corresponding to a given $\mathrm{F}_{\mathrm{D}}$. To keep the maximum total field below the critical field, the largest practical $B_{0}=0.067 \mathrm{~T}$. For $F_{D}=2.15 \times 10^{-4} \mathrm{~N}$, this value of $B_{0}$ corresponds to the field gradient $\mathrm{dB} / \mathrm{dz}=0.742 \mathrm{~T} / \mathrm{m}$ at the point of maximum displacement. The maximum field experienced by the sphere due to the drag coils is $B_{0, \max }=1.5 \cdot \mathrm{B}_{0}=0.101 \mathrm{~T}$. Note that one may still adjust the stiffness of the field, since $d B / d z \sim C_{2} \cdot \delta$, where $C_{2}$ is a constant. A measurable signal is generated in the sensing coils for $\delta_{\mathrm{MAX}}=10 \mathrm{~mm}$, corresponding to $\mathrm{F}_{\mathrm{D}}=2.15 \times 10^{-4} \mathrm{~N}$. 


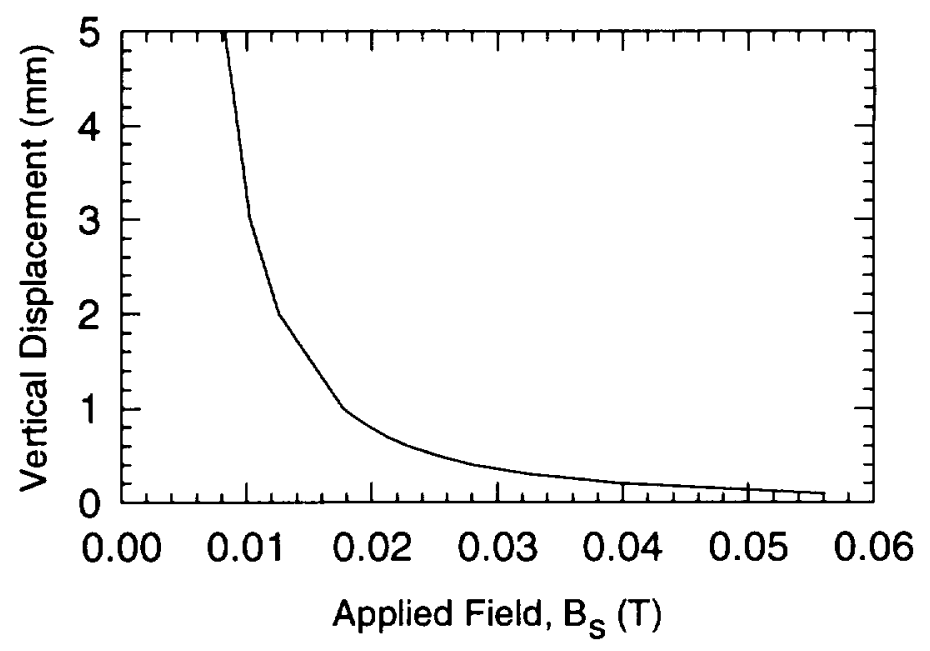

Figure 2. Support field stiffness. The sphere displaces slightly in the support field under the influence of its weight. The maximum field experienced by the sphere should remain well below the critical field for the niobium coating. For $\mathrm{B}_{\mathrm{s}}=0.018 \mathrm{~T}$, the support coils should be offset $1 \mathrm{~mm}$ with respect to the test section in order for the sphere to remain centered in the flow field.

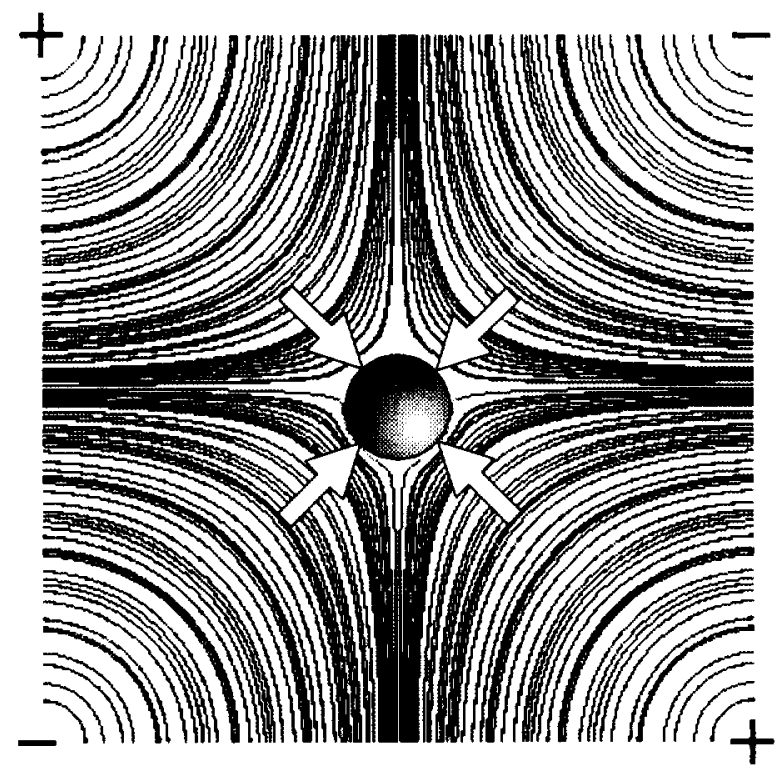

Figure 3. Field lines in the quadrapole support system. Arrows indicate regions of highest field intensity. Note the slight asymmetry due to the vertical displacement of the sphere under the influence of its own weight. (courtesy, Soren Prestemon, NHMFL/FSU) 


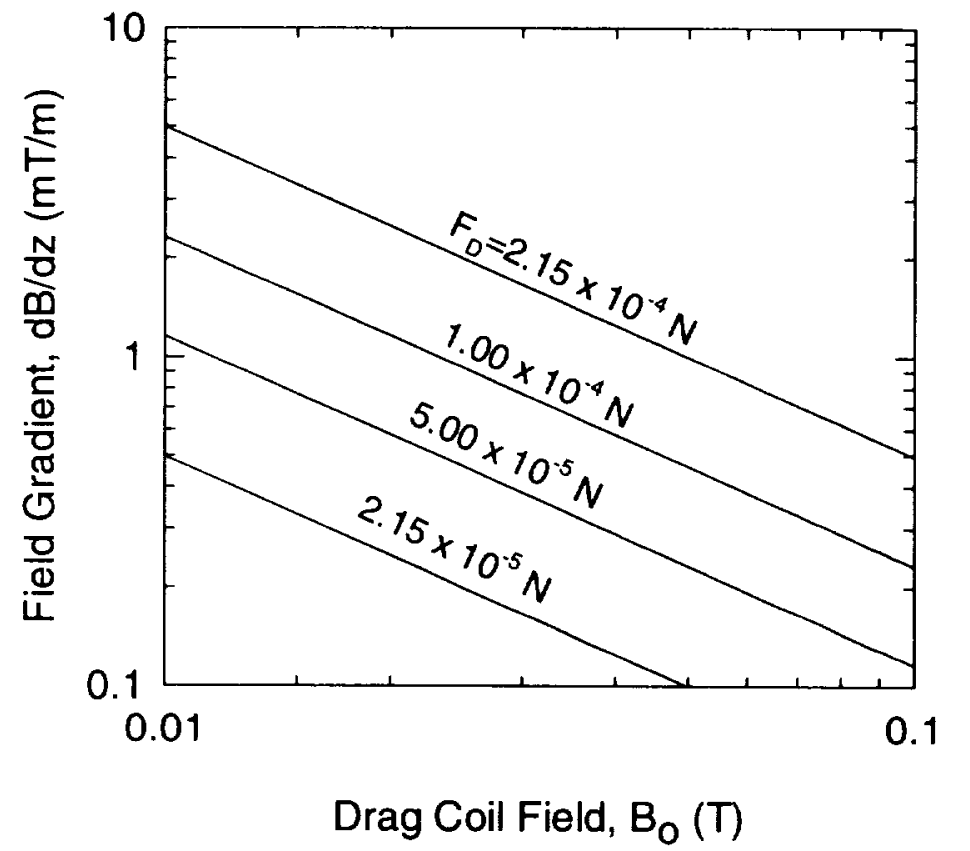

Figure 4. Drag coil field gradient vs. applied field $\mathrm{B}_{0}$. The optimum signal for drag measurement is achieved for $\mathrm{dB} / \mathrm{dz}$ as small as possible for a particular drag, while remaining well below the critical field $B_{c} \sim 0.15 T$.

\section{DRAG MEASUREMENT SCHEMES}

The proposed drag measurement is accomplished by allowing the sphere to deflect a small amount $\delta$, under applied drag. Sensor coils, placed as illustrated in Figures 1 and 5 , will then detect a flux change $\delta \phi$. These sensor coils should be counterwound to increase the flux change through the circuit, as well as to decrease the total inductance of the sensor coil pair. Counterwinding also makes the system insensitive to stray field fluctuations in the laboratory. The flux change detected by two single loops placed $2 \Delta$ apart and having a radius $R_{1}$ is

$$
\delta \phi=\pi d_{s}^{3} B_{0} R_{1}^{2}\left(R_{1}^{2}+\Delta^{2}\right)^{-3 / 2}\left\{\left[1+\frac{\delta(2 \Delta+\delta)}{R_{1}^{2}+\Delta^{2}}\right]^{-3 / 2}-\left[1+\frac{\delta(-2 \Delta+\delta)}{R_{1}^{2}+\Delta^{2}}\right]^{-3 / 2}\right\} .
$$

Figure 6 plots this equation as $\delta \phi / \mathrm{B}_{0}$ vs. $2 \Delta$ for different values of $\delta$. For small $\delta$, the optimum flux change occurs for $2 \Delta=\mathbf{R}_{1}$. For the experiment proposed here, $R_{1}=30 \mathrm{~mm}$. An approximate expression for this optimum flux is shown in Equation 5, underlining the linear dependence of $\delta \phi$ upon $\delta$ for small displacements. 


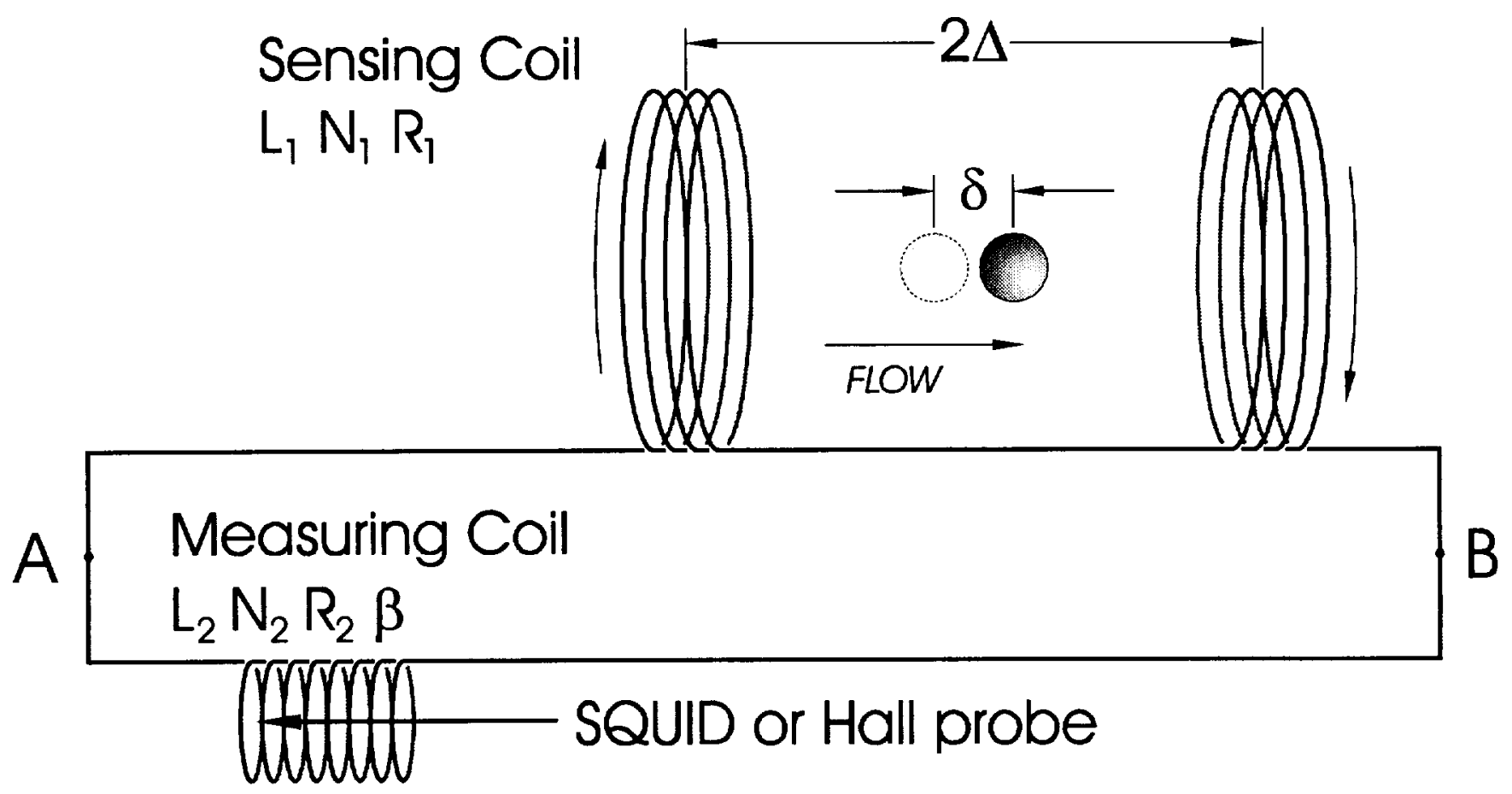

Figure 5. Drag measurement scheme. In the passive magnetic drag measurement, the sphere is allowed to deflect slightly under applied drag. This results in change in the magnetic flux contained by the superconducting sensing coils, which in turn induces current flow in the closed superconducting loop. The current flow creates a field in the secondary, or measuring coil which may be measured by several methods.

$$
\delta \phi_{\text {MAX }}\left(\text { at } R_{1}=2 \Delta\right)=5.3950 \frac{d_{s}^{3} B_{o} \delta}{R_{1}^{2}}
$$

The changing flux $\delta \phi / \mathrm{dt}$ induces a voltage, which can in principle be measured across open connections as $A$ and $B$ in Figure 5. The total flux change then follows from integrating $V_{A B}(t)$. Since $V_{A B}(t)$ depends upon the rate at which the sphere responds to changing flow conditions however, displacements which evolve over 10 seconds or more may produce signals too small to record. One needs a measurement scheme which depends only on the distance $\delta$, independent of the response time; in effect, the perfect integrator. Such performance may be achieved via a closed superconducting circuit, as illustrated in Figure 5. The secondary, or measuring coil may be either a small solenoid wrapped around a hall probe, or a SQUID (Superconducting QUantum Interference Device) to measure the current induced in the circuit. In either case, the flux in the circuit is conserved, so that

and

$$
N_{1}\left(\delta \phi+\phi_{1}\right)+N_{2} \phi_{2}=0
$$

$$
\frac{N_{1} \phi_{1}}{L_{1}}=\frac{N_{2} \phi_{2}}{L_{2}}=I
$$




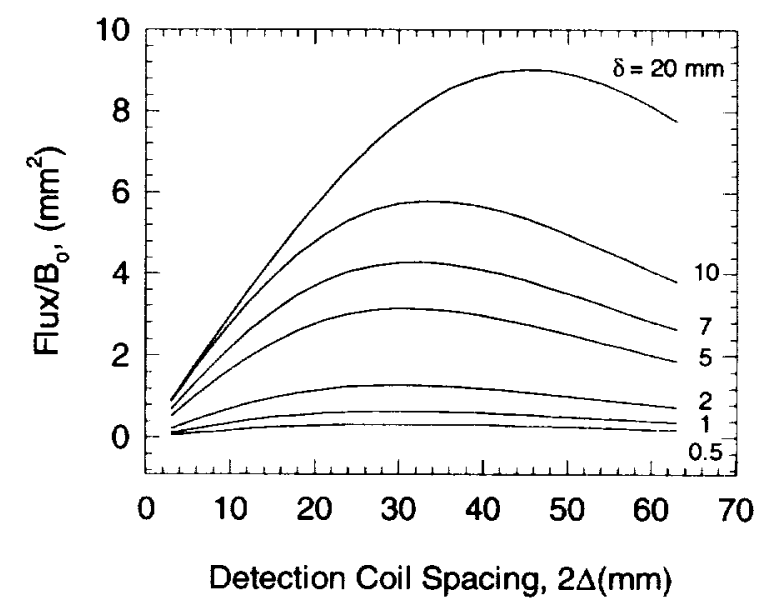

Figure 6. Measured flux vs. detection coil spacing. The optimum flux change occurs for a coil spacing $2 \Delta=R_{1}$. A greater signal also results from allowing a larger deflection $\delta$ for a given drag $\mathrm{F}_{\mathrm{D}}$. This is also apparent in Equation 10.

where $N_{1}$ is the number of turns/loop in the sensing coil, and $N_{2}$ is the number of turns in the measuring coil. Thus, $N_{1}\left(\phi_{1}+\delta \phi\right)$ and $N_{2} \phi_{2}$ are the fluxes linked by the turns of the sensing and measuring coils, respectively. The inductance $L_{1}$ is the total inductance of the sensing coils, including mutual inductance. We now consider the two measurement options in greater detail.

\section{Drag Measurement Using a Hall Probe}

From Equations 6 and 7, the total flux in the measurement coil is

$$
N_{2} \phi_{2}=\frac{N_{1} \delta \phi}{1+\frac{L_{1}}{L_{2}}}
$$

where the hall probe records approximately $B=\phi_{2} \pi R_{2}{ }^{2}$. The maximum field $B$ is obtained when $L_{1}=L_{2}$, so that

$$
\mu_{o} R_{1} N_{1}^{2} f_{1}=\mu_{o} R_{2} N_{2}^{2} f_{2}
$$

where $f_{1}$ and $f_{2}$ are inductance shape factors. For particular $R_{1}$ and $R_{2}$, this effectively constrains the ratio of turns $\mathrm{N}_{1} / \mathrm{N}_{2}$. Figure 7 shows $B / B_{0}$ plotted against $2 \Delta$ for different values of $\delta$, with $R_{2}=1 \mathrm{~mm}$. It should come as no surprise to the reader that the optimum measured field occurs for approximately $2 \Delta=R_{1}$. For this coil spacing, the measured field has an analytic form: 


$$
B=\frac{0.21446 \mu_{o}}{\sqrt{\beta^{2}+1}} \frac{F_{D}}{d B / d z} \frac{\delta}{\sqrt{f_{1} f_{2}}} \frac{1}{R_{1}^{2.5} R_{2}^{1.5}}
$$

where $\beta=l / 2 \mathrm{R}_{2}$ is an aspect ratio for the measuring solenoid. Larger values of $\beta$ yield more uniform fields in the cross-section, however $\beta=1$ results in only a $15 \%$ variation in $B$ across the area of the solenoid (Hall probe), which we deemed an acceptable price for a larger nominal value of $B$. This was the value used in generating Figure 7. The optimal signal is achieved for $R_{2}, \beta$, and $d B / d z$ as small as possible, while allowing a large displacement $\delta$ for a given drag $F_{D}$. Note once again, that $B$ varies linearly with the drag.

For $\beta=1, R_{2}=1 \mathrm{~mm}$, and $\delta=10 \mathrm{~mm}$, the maximum $B / B_{0}=7.69 \times 10^{-2}$, corresponding to the peak drag $F_{D}=2.15 \times 10^{-4} \mathrm{~N}$. The experimentalist then realizes a maximum signal $B_{M A X}=B_{0} \cdot 7.69 \times 10^{-2}=50$ gauss. For $R_{1}=30 \mathrm{~mm}$ (fixed by the physical dimensions of the test section), and $R_{2}=1 \mathrm{~mm} \mathrm{~N} / \mathrm{N}_{1}=15.32$, so that $N_{1}=3$ allows a nearly optimum $\mathrm{N}_{2}=46$. Axial Hall probes this size are readily available with sensitivities of order $0.01 \mathrm{mV} / g a u s s$. Thus, we expect an unamplified maximum output of $0.5 \mathrm{mV}$. Such measurements are within the reach of the experimentalist, though one could wish for better resolution of small drag forces.

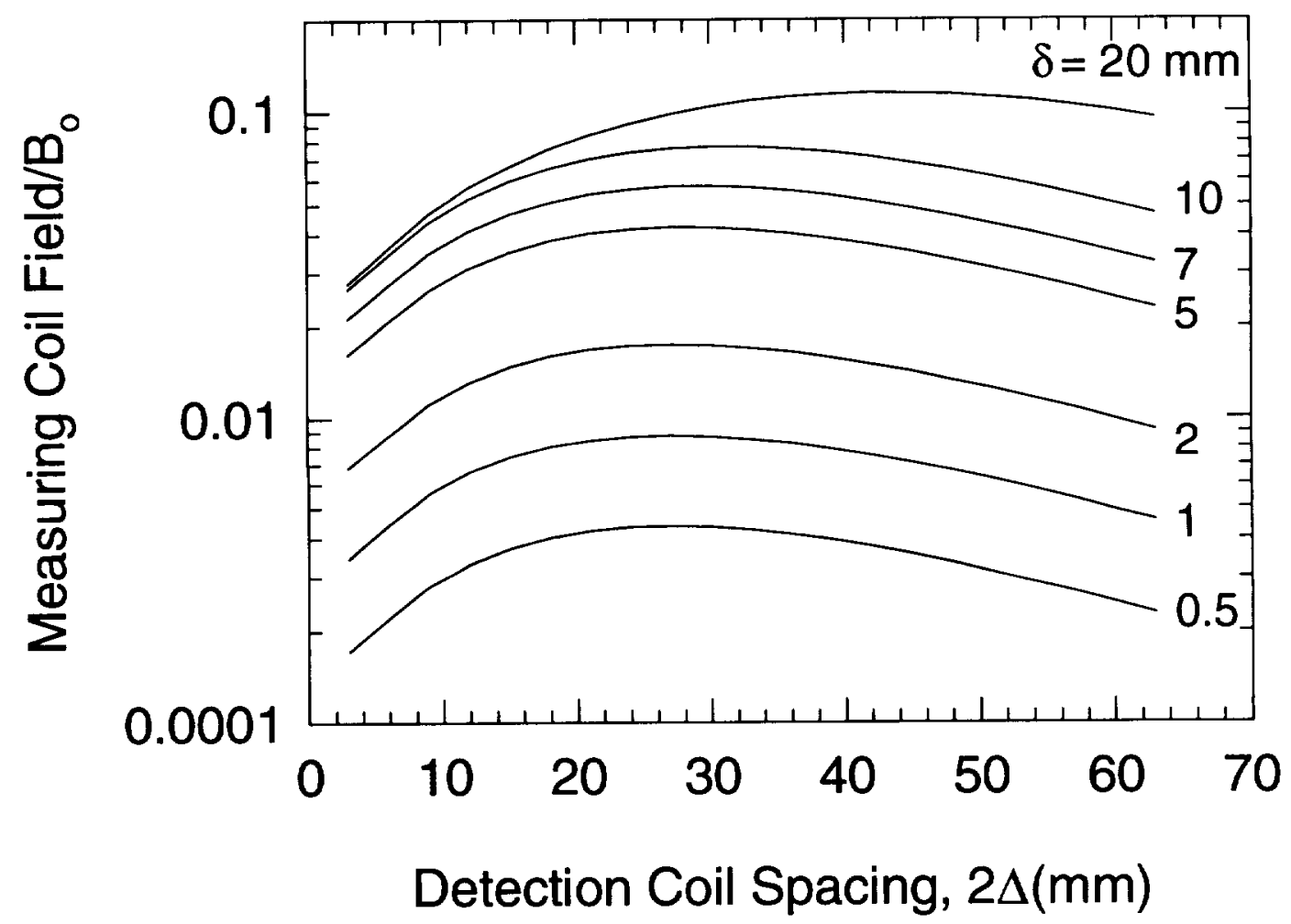

Figure 7. Induced field in the measuring coil vs. detection coil spacing. The maximum field measured by the Hall probe, $B_{\text {MAX }} \sim 50$ gauss, is achieved for $\beta=1, R_{2}=1 \mathrm{~mm}$, and $\delta=10 \mathrm{~mm}$, corresponding to a maximum drag $F_{D}=2.15 \times 10^{-4} \mathrm{~N}$. Again, the optimal spacing is $2 \Delta=R_{1}$. 


\section{Drag Measurement Using a Squid}

For greater resolution in measuring small drag forces, one may use SQUID technology. The closed superconducting circuit of Figure 5 appears the same, but the SQUID provides a sensitive measurement of the current. This current is expressed using Equations 6 and 7 as

$$
I=\frac{N_{1} \delta \phi}{L_{1}+L_{2}} \approx \frac{N_{1} \delta \phi}{L_{1}},
$$

where the approximation holds for small $\mathrm{L}_{1}>>\mathrm{L}_{2}$. In fact, a SQUID has too much sensitivity for use with the optimized field gradient discussed above. This is compensated for by building a much stiffer drag field, with smaller $B_{0}$ (equivalently, a much larger gradient $d B / d z$ ). Additionally, the circuit is 'de-tuned' such that $\mathrm{L}_{1} \gg \mathrm{L}_{2}$. Our SQUID has a maximum continuous range of $0.1-50 \mu \mathrm{A}$ with $0.1 \mu \mathrm{A}$ resolution over the entire span, and $L_{2}=2 \mu \mathrm{H}$. Using these values, Figure 8 plots $\mathrm{N}_{1} / \mathrm{B}_{0}$ against coil spacing $2 \Delta$, for various $\delta$. Though one may be tempted to select a large coil spacing, this tends to de-couple the coils from the sphere, as well as increase the noise from stray field fluctuations. With $2 \Delta=15 \mathrm{~mm}, \mathrm{~B}_{0}=0.01 \mathrm{~T}$, and a stiff field (say, $\delta=0.5 \mathrm{~mm}$ for $F_{D}=2.15 \times 10^{-4} \mathrm{~N}$ ), $\mathrm{N}_{1}=300$ turns/loop is sufficient to limit the maximum signal to $50 \mu \mathrm{A}$. Such a system will reliably resolve drag forces on the sphere as small as $F_{D}=4.3 \mu \mathrm{N}$.

Although the pickup coils are counterwound in an attempt to eliminate noise form stray fields in the laboratory, the sensitive nature of the SQUID requires additional shielding of all wires, coils, and electronics. It may even be necessary to build a superconducting shield around the entire experiment.

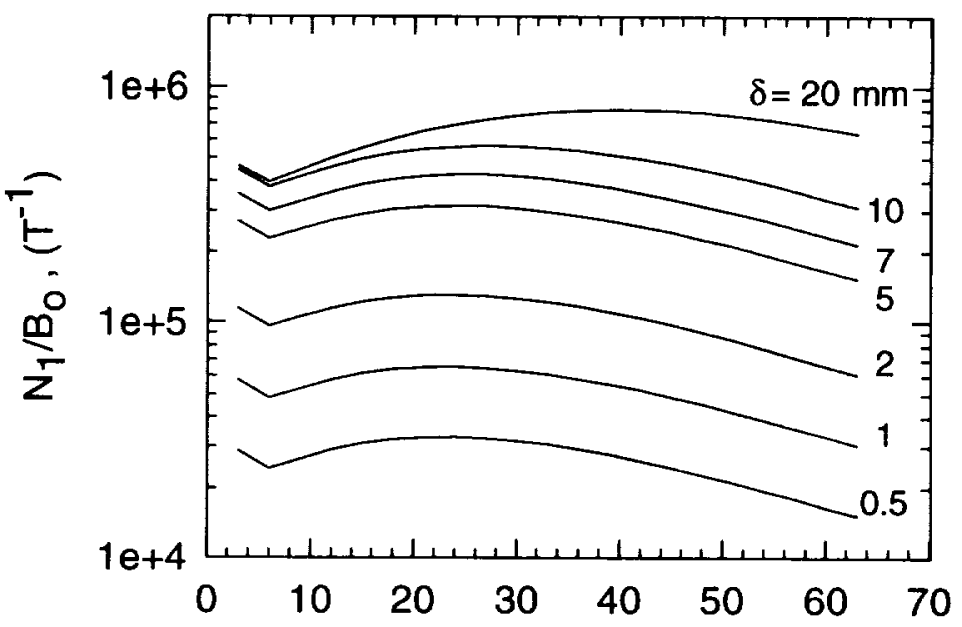

Detection Coil Spacing, $2 \Delta(\mathrm{mm})$

Figure 8. Turns/loop in the sensing coil to 'de-tune' the sensing circuit for use with a SQUID. For a stiff field gradient, $B_{0}=0.01 \mathrm{~T}$ (allowing a maximum $\delta=0.5 \mathrm{~mm}$ ) and $\mathrm{N}_{1}=300$ turns/loop limits the maximum induced current to the $50 \mu \mathrm{A}$ required for our SQUID. 


\section{PRACTICAL CONSIDERATIONS AND CONCLUSION}

In evaluating the different options and possible configurations for a superconducting MSBS, we have tried to keep in mind the practical implications on building and operating such an experiment. We conclude with a brief discussion of two of these; sphere position management, and calibration. The vertical orientation initially considered had impractical consequences for both of these.

In the horizontal orientation, calibration of the superconducting MSBS is not difficult. By carefully weighing the sphere initially, one may then tilt the experiment slightly out of horizontal with the MSBS energized, so that the weight has some small component directed along the tunnel. In the absence of flow, this becomes the only force measured by the MSBS. For a $1 \mathrm{~m}$ long test section in which a $0.01 \mathrm{~N}$ sphere is suspended, raising the upstream end by $21.5 \mathrm{~mm}(0.846 \mathrm{in})$ with respect to the outlet results in $2.15 \times 10^{-4} \mathrm{~N}$ resolved along the drag direction.

The issue of sphere position management is more complicated. Due to the vacuum and thermal isolation required for a liquid helium experiment, the sphere must be sealed inside the apparatus as it is assembled at room temperature, limiting access during the course of the experiment. Since the superconducting MSBS does not function above the critical temperature, the sphere must rest within some mounting or controlled position upon the lower surface of the tunnel while the experiment is inserted in the cryostat and cooled down. If the sphere somehow becomes displaced from this initial or rest position, it must relocate itself. Furthermore, this relocation process must be robust, since access is limited. This is especially true when the apparatus reaches its operating temperature. Finally, all of this must be accomplished so as not to disturb the flow. One promising solution is to construct the test section with walls bowed out very slightly, as with a slight conical taper extending up and down stream from the sphere's neutral position (its position in the center of the tunnel with the MSBS energized and without flow). This preserves the axisymmetric nature of the flow, and if done carefully may act to correct somewhat the blockage effects. With the walls bowed out slightly and the sphere resting upon the lower surface, there is a low-point on the tunnel wall directly adjacent to the neutral position, to which the sphere will settle. With the experiment cold, ramping the current up from zero lifts the ball gently from its rest position to the neutral position in the center of the tunnel.

As previously mentioned, once such an experiment is cold, access to the test section is limited. Thus, one should plan for every contingency, particularly the possibility of blow-by. One must have a passive, non-destructive, non-intrusive mechanism in place to intercept the sphere and return it to its rest position. One option would be to place a large-mesh fiberglass screen several inches downstream of the sphere's neutral/rest position. In the event of blow-by, the screen intercepts the sphere without scratching (damaging the $\mathrm{Nb}$ film). The MSBS may then be de-energized, allowing the sphere to roll along the lower tunnel wall back to the rest position.

In the present paper, we describe the theoretical and practical issues associated with designing, building and operating a superconducting MSBS for a liquid helium wind tunnel. Such a system could be developed as part of a modest effort in high Reynolds liquid helium flow testing. The approach is most easily applied for the suspension of symmetrical bodies, but with further modifications could be extended to asymmetrical objects where active control may be incorporated. With such an effort it would be possible to develop much of the technology necessary to apply magnetic suspension systems in large scale liquid helium flow facilities which could be utilized for a variety of studies at very high Reynolds number $\left(\operatorname{Re}>10^{6}\right)$. 


\section{ACKNOWLEDGMENTS}

We wish to acknowledge the support of the Naval Undersea Warfare Center under contract number N66604-96-C-A093. We also wish to acknowledge partial support from the National High Magnetic Field Laboratory at Florida State University. Finally, we would like to thank Soren Prestemon for his work in plotting the magnetic field lines for the quadrapole field shown in Figure 3.

\section{REFERENCES}

1. Pope, A.; and Harper, J. J.: Low Speed Wind Tunnel Testing. John Wiley and Sons, New York, 1966 [Second Edition: Rae, W. H.; and Pope, A.: John Wiley and Sons, 1984].

2. Goodyear, M. J. in High Reynolds Number Flows Using Liquid and Gaseous Helium, R. J. Donnelly, ed.; Springer-Verlag, New York, 1991, pp. 131-152.

3. Lawing, P. L. in High Reynolds Number Flows Using Liquid and Gaseous Helium, R. J. Donnelly, ed.; Springer-Verlag, New York, 1991, pp. 153-164.

4. Britcher, C. P. in High Reynolds Number Flows Using Liquid and Gaseous Helium, R. J. Donnelly, ed.; Springer-Verlag, New York, 1991, pp. 165-180.

5. Donnelly, R. J., ed.: High Reynolds Number Flows Using Liquid and Gaseous Helium. Springer-Verlag, New York, 1991, pp. 131-152. 